MATEMATIKA, 2017, Volume 33, Number 1, 113-118

(C) Penerbit UTM Press. All rights reserved

\title{
Some new theoretical work on Half Butterfly Method for Hamiltonian circuit in complete graph
}

\author{
${ }^{1}$ Maizon Mohd Darus, ${ }^{2}$ Haslinda Ibrahim and ${ }^{3}$ Sharmila Karim \\ ${ }^{1,2,3}$ Department of Mathematics and Statistics, School of Quantitative Siences \\ College of Arts and Sciences, Universiti Utara Malaysia, \\ 06010 Sintok, Kedah, MALAYSIA \\ e-mail: ${ }^{1}$ maizonmohddarus@gmail.com, ${ }^{2}$ linda@uum.edu.my, ${ }^{3}$ mila@uum.edu.my
}

\begin{abstract}
A new method to construct the distinct Hamiltonian circuits in complete graphs is called Half Butterfly Method. The Half Butterfly Method used the concept of isomorphism in developing the distinct Hamiltonian circuits. Thus some theoretical works are presented throughout developing this method.
\end{abstract}

Keywords Complete graphs; decomposition; Hamiltonian circuits; Half Butterfly Method.

2010 Mathematics Subject Classification 05E15, 05E40

\section{Introduction}

Graph decomposition regarding complete graph has attracted the researchers [1-4]. Among these, complete graph decomposition into Hamiltonian circuit (HC) is attention-grabbing due to its vast advantages such as analyzing interconnection network in multicomputer [5], privacy data mining [6], butterfly network [7], and DNA physical mapping [8].

Even though several fast algorithms have been discovered [9-12], the geometric representations of the $\mathrm{HC}$ have not been presented. This representation is vital to present the circuits directly. Therefore, the similarities or differences among the circuits can be perceived. Thus the new method namely Half Butterfly Method (HBM) is introduced by Maizon [13] to fill the gap. The HBM was inspired by [14] as they introduced Butterfly Strategy (BS) to solve complete bipartite graph $K_{n, n}$ decomposition into one factor. The detail of geometric representation of $\mathrm{HC}$ using $\mathrm{HBM}$ can be found from [15].

In this paper, we aim to present the new theoretical work on HBM novel method to construct the distinct $\mathrm{HC}$ in complete graph decomposition. We start with some basic terminologies and definitions needed along this paper.

\section{Basic terminologies and definitions}

We follow standard graph theory notations. A graph $G$ consists of a set of vertices $V(G)$ together with a set of edges $E(G)$. A complete graph with $n$ vertices $K_{n}$ is a simple undirected graph where each vertex is adjacent to all the other vertices. A Hamiltonian circuit $(\mathrm{HC})$ is a circuit that starts and ends at the same vertex, and passes through every vertex exactly once.

Definition 1 Let $G$ and $H$ be two complete graphs. Suppose the sets of vertices

$$
\left\{x_{1}, x_{2}, x_{3}, \ldots, x_{n}\right\} \in G \text { and }\left\{x_{a}, x_{b}, x_{c}, \ldots, x_{z}\right\} \in H,
$$


where $1 \leq a, b, c, z \leq n$. A function

$$
g=\left(\begin{array}{ccccc}
x_{1} & x_{2} & x_{3} & \ldots & x_{n} \\
x_{a} & x_{b} & x_{c} & \ldots & x_{z}
\end{array}\right)
$$

maps the vertices $\left\{x_{1}, x_{2}, x_{3}, \ldots, x_{n}\right\}$ of $G$ to other vertices $\left\{x_{a}, x_{b}, x_{c}, \ldots, x_{z}\right\}$ of $H$ where $\left\{x_{a}, x_{b}, x_{c}, \ldots, x_{z}\right\}$ are the images for every element in $G$. That is, $x_{1} \rightarrow x_{a}, x_{2} \rightarrow x_{b} \ldots$, $x_{n} \rightarrow x_{z}$ for $n \in Z^{+}$. Then, the mapping is written as a product of transposition

$$
\left(x_{1}, x_{a}\right)\left(x_{2}, x_{b}\right) \ldots\left(x_{n}, x_{z}\right) .
$$

Definition 2 Let $A$ and $B$ be two circuits with $n$ vertices. If the mapping of $A$ and $B$ is $(1, a)(2, b)(3, c) \ldots(n, z)$ and $(z, n) \ldots(c, 3)(b, 2)(a, 1)$ respectively, where $1 \leq a, b, c, z \leq n$, then $A$ and $B$ has an opposite mapping.

Definition 3 Let $A$ be a circuit with direction $\left(x_{1}, x_{2}, x_{3}, \ldots, x_{n-1}, x_{n}, x_{1}\right)$. Then, a circuit $B$ is a mirror image to circuit $A$ if the direction of $B$ is $\left(x_{1}, x_{2}, x_{3}, \ldots, x_{n-1}, x_{n}, x_{1}\right)$.

Definition 4 Let $A$ and $B$ be two circuits with $n$ vertices. $A$ is isomorphic to $B$ when $B$ is the mirror image of $A$.

$\mathrm{HBM}$ is a method to decompose $K_{n}$ into distinct $\mathrm{HC}$ with different path. HBM consist four steps. The first step is creating direction, second step is fix and shift every vertex of the direction obtained in first step. Meanwhile the next step is finding the mapping and final step is drawing the HC. The example of construction can found in [13]. Thus in next section, we discuss the theoretical work on HBM.

\section{Some theoretical concepts on HBM}

Our proposed HBM yielded the following lemmas, propositions, and theorems.

Lemma 1 There are $(n-1)$ blocks to create directions for all $K_{n}, n \geq 3$.

Proof Suppose $\left\{x_{1}, x_{2}, x_{3}, x_{4}, \ldots, x_{n}\right\}$ are vertices of $K_{n}$. Let $\lambda_{1}$ be block $1, \lambda_{2}$ be block $2, \lambda_{3}$ be block 3 , and $\lambda_{m}$ be the last block. In generating the directions, in each block, we have the following situation:

$\lambda_{1}$ : Vertex $x_{1}$ is fixed as the starting location and $x_{2}$ is fixed to be the second location for the direction.

$\lambda_{2}$ : Vertex $x_{1}$ is fixed as the starting location and $x_{3}$ is fixed to be the second location for the direction.

$\lambda_{3}$ : Vertex $x_{1}$ is fixed as the starting location and $x_{4}$ is fixed to be the second location for the direction.

$\lambda_{m}$ : Vertex $x_{1}$ is fixed as the starting location and $x_{n}$ is fixed to be the second location for the direction.

For each block, a vertex (vertex $x_{1}$ ) is fixed to be the starting location. Then, there are $(n-1)$ vertices left to be chosen as the second location, consecutively, in each block. Since

there are $(n-1)$ vertices to be the second location, then there are $(n-1)$ blocks to create direction for all $K_{n}, n \geq 3$. 
Lemma 2 There are $(n-2)$ directions in each block.

Proof. By Lemma 1 , there are $(n-1)$ blocks to create the directions. In each block, two vertices are fixed to be the starting location and the second location. Since there are $n$ vertices, and two vertices are fixed such that vertex $x_{i}$ and vertex $x_{j}$ for $j>i$, then there are $(n-2)$ vertices left to be the third location for each direction. Thus, $(n-2)$ directions are obtained in each block.

Lemma 1 and Lemma 2 provide the total number of directions and blocks for a complete graph. The following propositions and theorems have been provided based on Lemma 1 and Lemma 2.

Proposition 3 The total number of directions that could be created in $K_{n}, n \geq 3$ is ( $n-$ 1) $(n-2)$.

Proof. From Lemma 1, let $\lambda_{k}$ be the blocks in creating the directions, for $1 \leq k \leq n-1$. Since we have $(n-1)$ blocks and each block has $(n-2)$ directions, thus we have

$$
\sum_{k=1}^{n-1} \lambda_{k}=(n-2)(n-1)
$$

directions in $K_{n}$.

In other way, we can prove this theorem using the idea of arithmetic sequences as discussed below. From Lemmas 1 and 2, we have the total number of directions for each block as shown below.

$$
\begin{aligned}
\lambda_{1} & =(n-2) \text { directions } \\
\lambda_{1}+\lambda_{2} & =(n-2)+(n-2)=2(n-2) \\
& =(2 n-4) \text { direction } \\
\lambda_{1}+\lambda_{2}+\lambda_{3} & =(n-2)+(n-2)+(n-2)=3(n-2) \\
& =(3 n-6) \text { direction } \\
\lambda_{1}+\lambda_{2}+\lambda_{3}+\lambda_{4} & =(n-2)+(n-2)+(n-2)+(n-2)=4(n-2) \\
& =(4 n-8) \text { direction } \\
\lambda_{1}+\lambda_{2}+\lambda_{3}+\lambda_{4}+\lambda_{5} & =(n-2)+(n-2)+(n-2)+(n-2)+(n-2)=5(n-2) \\
& =(5 n-10) \text { direction }
\end{aligned}
$$

We transform the above results into arithmetic sequence as presented below. Let $A_{k}$ be the arithmetic sequence and $a_{k}$ denotes the elements in $A_{k}$ for $1 \leq k \leq n$. Then

$$
A_{k}=\left\{n-2,2 n-4,3 n-6,4 n-8,5 n-10, \ldots, a_{k}\right\},
$$

where $a_{1}=n-2, a_{2}=2 n-4, a_{3}=3 n-6, \ldots a_{k}$. To find the last term of $A_{k}$, we consider the formula

$$
a_{k}=a_{1}+(k-1) d
$$

where $a_{1}$ is the first element of $A_{k}, d$ is the common difference and $k$ is the number of the element to find. To determine the common difference, $d$ we use

$$
d=a_{i}-a_{j}
$$

where $i=j+1$ and $1 \leq i<j \leq k$. Then, by taking any element of $A_{k}$ in (1.1), we have 


$$
d=n-2 .
$$

Here, we take $k=n-1$ due to Lemma 1. Therefore, from (1.2), (1.3) and (1.4), we have

$$
\begin{aligned}
a_{k} & =a_{1}+(k-1) d \\
a_{n-1} & =a_{1}+((n-1)-1) d=(n-2)+((n-1)-1)(n-2) \\
& =(n-2)+(n-2)(n-2)=n^{2}-3 n+2=(n-2)(n-1)
\end{aligned}
$$

Thus, the last element of $A_{k}$ is $[(n-2)(n-1)]^{\text {th }}$ element, which gives the total number of directions that can be produced for every $K_{n}$ for $n \geq 3$.

Proposition 4 There are $n(n-1)$ Hamiltonian circuits from each direction in $K_{n}, n \geq 3$.

Proof From Proposition 3, there are $(n-1)(n-2)$ directions in $K_{n}$. The vertices $x_{1}, x_{2}, x_{3}, \ldots, x_{n}$ in each direction are fixed-and-shifted, where one vertex is fixed and the remaining vertices are shifted to the left.

Suppose the first direction of $K_{n}$ is $\alpha_{1}=\left\{x_{1}, x_{2}, x_{3}, x_{4}, \ldots, x_{n}\right\}$. When each vertex of $\alpha_{1}$ is fixed, then we have $(n-1)$ vertices left to be shifted to the left. Since there are $n$ vertices to be fixed, then we have

$$
\sum_{i=1}^{n}(n-1)=\underbrace{(n-1)+(n-1)+(n-1)+\ldots+(n-1)}_{n \text { times }}=n(n-1)
$$

Hamiltonian circuits with similar paths from each direction in $K_{n}$.

Proposition 5 There are

$$
\frac{(n-1)(n !)}{(n-3) !}
$$

Hamiltonian circuits from all directions in $K_{n}, n \geq 3$.

Proof For every $K_{n},(n-1)(n-2)$ directions are obtained (Proposition 3$)$. For each direction, when one vertex is fixed, there are $(n-1)$ vertices left to be shifted to the left. Since there are $n$ vertices to fix, then we have

$$
\begin{aligned}
\sum_{j=1}^{n} \sum_{i=1}^{n-1} & (n-2)(n-1)=\sum_{i=1}^{n-1}[\underbrace{(n-2)(n-1)+(n-2)(n-1)+\ldots+(n-2)(n-1)}_{n \text { times }}] \\
& =\sum_{i=1}^{n-1}(n)(n-2)(n-1) \\
& =\underbrace{(n)(n-2)(n-1)+(n)(n-2)(n-1)+\ldots+(n)(n-2)(n-1)(n-1)}_{(n-1) \text { times }} \\
& =(n-1)(n)(n-2)(n-1)=(n-1)[(n-2)(n-1)(n)] \\
& =(n-1)\left[\frac{n !}{(n-3) !}\right]=\left[\frac{(n-1)(n !)}{(n-3) !}\right]
\end{aligned}
$$

circuits from all directions in $K_{n}$.

Remark The Hamiltonian circuits produced from all directions in $K_{n}$ (Proposition 5 ) are inclusive of duplicate circuits, i.e. circuits with similar mapping and circuits with opposite mapping. Thus, the mapping of these circuits will be used for the elimination purposes. Therefore, Definition 1 are followed to eliminate the similar mappings and opposite mappings in order to get the distinct circuits. 
Theorem 6 Let $G$ be a complete graph. Then $G$ is decomposable into $\frac{1}{2}(n-1)$ ! distinct Hamiltonian circuits with different paths for all $n \geq 3$.

Proof. Suppose $G$ is a $K_{n}$, then there exist $n$ vertices. The total number of Hamiltonian circuits of $K_{n}$ is known to be $n$ !. Since there are $n$ vertices, then there are $n$ vertices available as starting locations. Thus, $K_{n}$ has $\frac{n !}{n}$ Hamiltonian circuits with similar paths. Based on Definition 4 and Lemma 1, we have

$$
\begin{aligned}
& \frac{n !}{n}-\frac{(n-1) !}{2} \\
& =\frac{2 n !-n(n-1) !}{2 n} \\
& =\frac{2 n !-n !}{2 n} \\
& =\frac{n !}{2 n} \\
& =\frac{(n-1) !}{2}
\end{aligned}
$$

distinct Hamiltonian circuits with different paths from $K_{n}$.

\section{Conclusion}

This paper presented the theoretical works on HBM. Our future research is we will attempt to apply this HBM for the number of different structure $\mathrm{HC}$ from $K_{n}$.

\section{Acknowledgement}

The authors gratefully acknowledged the financial support received in the form of a High Impact Group Research Grant (PBIT) (Code: 12621) from Universiti Utara Malaysia.

\section{References}

[1] Anitha, R. and Lekshmi, R. S. N-Sun decomposition of complete, complete bipartite and some harary graphs. International Journal of Mathematics Sciences. 2008. 2(1): 33-38.

[2] Brualdi, R. A. and Schroeder, M. W. Symmetric Hamilton cycle decompositions of complete graphs minus a 1-factor. Journal of Combinatorial Designs. 2011. 19(1): 115.

[3] Gyarfas, A., Ruszinko, M., Sarkozy, G. N. and Szemeredi, E. Partitioning 3-coloured complete graphs into three monochromatic cycles. The Electronic Journal of Combinatorics. 2011. 18: P53.

[4] Kaski, P. and Ostergard, P. R. J. There are 1, 132, 835, 421, 602, 062, 347 nonisomorphic one-factorizations of $K_{14}$. Journal of Combinatorial Designs. 2009. 17(2): 147-159. 
[5] Choi, D., Lee, O. and Chung, I. A parallel routing algorithm on recursive cube of rings networks employing the Hamiltonian circuit Latin square. Information Sciences. 2008. 178(6): 1533-1541.

[6] Dong, R. and Kresman, R. Notes of privacy-preserving distributed mining and Hamilton Graphs into blown-up cycles $C_{m}$ [2]. Discrete Mathematics. 2010. 310(5): 1003-1015.

[7] Hwang, S. C. and Chen, G. H. Fault-free Hamiltonian cycles in faulty butterfly graphs. Paper presented at the Seventh International Conference on Parallel and Distributed Systems 2000. Iwate, Japan.

[8] Grebinski, V. Reconstructing a Hamiltonian cycle by querying the graph: Application to DNA physical mapping. Discrete Applied Mathematics. 1998. 88: 147-165.

[9] Dharwadker, A. A new algorithm for finding Hamiltonian circuits. Paper presented at the Proceedings of the Institute of Mathematics on December 2004. University of Alaska, Fairbanks.

[10] Riaz, K. and Khiyal, M. S. H. Finding Hamiltonian cycle in polynomial time. Information Technology Journal. 2006. 5(5): 851-859.

[11] Babar, G. M., Khiyal, S. H. and Saeed, A. Finding Hamilton circuit in a graph. Paper presented at the International Conference on Scientific Computing, CSC 2006. Las Vegas, Nevada, USA.

[12] Chalaturnyk, A. A Fast Algorithm for Finding Hamilton Cycles. University of Manitoba, Canada: Masters Dissertation. 2008.

[13] Darus, M. M. Geometric Representations of Distinct Hamiltonian Circuits in Complete Graph Decomposition. Universiti Utara Malaysia: Master Thesis. 2015.

[14] Gopal, A. P., Kothapalli, K., Venkaiah, V. C. and Subramaniam, C. R. Various one-factorizations of complete graphs. 2007 Technical Report at International Institute of Information Technology, Hyderabad, India. Retrieved from http://people.csail.mit.edu/prasant/factorizations.pdf. 2007, April 2.

[15] Darus, M. M., Ibrahim, H. amd Karim, S. The construction of distinct circuits of length six for complete graph K6. International Journal of Applied Mathematics and Statistics. 2015. 53(1): 17-31. 